Gebrauch. Bei Frühgeburten, die Menstruation befördernd und bei Blutspucken empfohlen.

(Fortsetzung folgt.)

\title{
Notiz über die Sammlung des Zibeths;
}

von

Dr. X. Landerer in Athen.

Von einem mir befreundeten Kaufmann, der sich in Zedda mit dem Handel und der Sammlung des Zibeths beschäftigt hatte, habe ich folgende Notiz darüber erhalten:

Alles stark Riechende, alles Nervenstärkende wird mit dem Namen Mosko bezeichnet; jedoch diese Secretionsflüssigkeit nennen die Orientalen Sebet, indem auch das Thier Sebet heisst.

Die Zibethkatze, Viverra Zibetha, ist in Hindostan, Siam und auf vielen Molukkischen Inseln einheimisch, findet sich jedoch auch in Nubien, Abyssinien und andern Plätzen Afrikas, wo sie des Zibeths wegen gejagt und gefangen wird, zu welchem Zweck man sie in Käfige einsperrt, und ihnen dort den Zibeth ausnimmt. Den durch Erschiessen erlegten Thieren wird das Zibeth sogleich nach erfolgtem Tode ausgenommen, was jedoch so gering ist, dass es sich kaum der Mühe lohnt. Da die Thiere sich dieses Secrets durch Anreiben an Bäumen zu entledigen pflegen, so kann man, durch den Geruch geleitet, dasselbe von den Baumstämmen abkratzen; dieser Zibeth ist jedoch mit einer Menge anderer vegetabilischer Stoffe vermengt, und auch die Jäger selbst vermischen denselben mit eingedampftem Weinmoste und Fett, um ihn im feuchten und schmierigen Zustande zu erhalten.

Haben nun diese Zibeth-Sammler eine hinreichende Menge gesammelt, so wird derselbe in grosse Ochsenhörner gefüllt und an die Kaufleute verkauft, welche ihn sehr theuer bezahlen, nämlich das Dramm (1 Quentchen) 
mit 6-10 Piaster. Einen höheren Werth besitzt derjenige Zibeth, der von den Leuten, welche die Thiere in Käfigen aufbewahren und füttern, gesammelt wird. Die selır wilden Katzen werden in grossen, sehr langen Käfigen, grossen Hühnerställen ähnlich, die aus eisernen Stangen gemacht sind, aufbewahrt, gewöhnlich je zwei in einem Kätig. Ihre Nahrung besteht in thierischen und vegetabilischen Stoffen, Fleisch, Fischen, Käse, Jagurt (d. i. saure Milch), Datteln. Unter den vegetabilischen Nahrungsstoffen soll bosonders die Frucht von Durio Zibethinus zu der Secretion des Zibeths beitragen, und durch dieselbe auch die Zibeththiere angelockt und gefangen werden. Werden sie in ihren Käfigen sehr unruhig und verrathen gegen die sich ihnen Nihernden cine grössere Beisslust, so wird der Zibeth ausgenommen, was auf folgende Weise geschieht. Mittelst eines Strickes, den man den Thieren um den Leib wirft, sucht man sie an die Gitter des Käfigs zu ziehen und nimmt sodann mit einem eisernen oder hölzernen Löffelchen den Zibeth aus der kleinen Tasche, die sich zwischen dem Anus und den Geschlechtstheilen befindet. Unmittelbar nach der Herausnahme hat die Flüssigkeit eine milchweisse Farbe und eine käseartige Consistenz, sie wird auf Kaffeetassen aufgestrichen und an der Sonne getrocknet, wodurch derselbe eine hellbraune Farbe annimmt, und in diesem noch schmierigen Zustande wird derselbe in kleine Blechbüchsen gefüllt und diese zugelöthet. Dieser Zibeth ist rein und kostet auch um das Doppelte mehr als der in den Hörnern vorkommende.

Von Nubien und Abyssinien kommt der Zibeth nach Smyrna und Konstantinopel und wird daselbst zu den mannigfaltigsten Wohlgeruichen, zu Pomaden, so wie auch zu Räucherungs-Gegenständen verwendet, ebenfalls zu Ilags, Heilmitteln, Mantsuns, Melhems u. s. w., welche nervenstärkende Eigenschaften besitzen. 